AN. MED. InTERNA (Madrid) Vol. 19, N. $^{\circ} 3$, pp. $143-150,2002$

\title{
Metabolismo óseo y pérdida de masa ósea en los trastornos de la alimentación
}

\author{
M. ESCALANTE BOLEAS, R. FRANCO VICARIO, V. BUSTAMANTE MURGA, \\ F. MIGUEL DE LA VILLA
}

Servicio de Medicina Interna. Hospital de Basurto. Bilbao. Bizcaia

\author{
BONE METABOLISM AND LOSS OF BONE MASS IN EATING \\ DISORDES
}

\section{RESUMEN}

Los trastornos de la alimentación (anorexia y bulimia nerviosa)afectan al 1-3\% de las mujeres jóvenes adultas. Edad tardía de inicio, duración de amenorrea e índice bajo de masa corporal son factores de riesgo de osteopenia en pacientes bulímicas.

Se produce desmineralización cuando la reabsorción sobrepasa a la formación ósea en la AN, lo que lleva al desarrollo de osteopenia, osteoporosis secundaria y mayor riesgo de fracturas patológicas.

La patofisiología de la desmineralización se atribuye a diversos factores como duración de la amenorrea, deficiente absorción de calcio, ejercicio físico extremo, déficit de $1,25(\mathrm{OH})$ vit $\mathrm{D}$, bajo aclaramiento de creatinina, exceso de cortisol sérico y urinario y niveles altos de GH.

Los tratamientos recomendados son rehabilitación nutricional (el más importante), suplementos de calcio, ejercicio moderado, terapia estrogénica cuando esté indicada, tratamiento antidepresivo, flúor en casos seleccionados, bifosfonatos e IGF-1 recombinante humano.

Un interrogatorio preciso es esencial en mujeres con peso ligeramente descendido y con transtornos menstruales.

PALABRAS CLAVE: Desmineralización. Anorexia-bulimia. Patofisiología. Tratamientos.

\begin{abstract}
Eating disorders (anorexia and bulimia nervosa) are present in 1$3 \%$ of young female women. A later beginning, prolonged amenorrhea and low body mass index are risk factors of osteopenia in bulimic patients.

Bone demineralization in anorexia nervosa is due to a greater resorption than bone formation leading to osteopenia, secondary osteo porosis and increased risk of pathologic fractures.

Pathophysiology of bone disease includes factors as long duration of amenorrhea, deficient absorption of calcium, extreme physical exercise, 1,25 vitamin D defficiency, low creatinine clearance, increased blood and urinary cortisol and high levels of GH. Proposed treatments are nutritio nal improvement and weight gain (the most important), calcium supple mentation, moderate exercise, estrogens, antidepressive drugs, fluoride in selected cases, byphosphonates and recombinant human IGF-1.

An adequate questionnary is essential in women with slightly decre ased weight and mentrual disorders.
\end{abstract}

KEY WORDS: Demineralization. Anorexia-bulimia. Pathophysiology. Treatments.

Escalante Boleas M, Franco Vicario R, Bustamante Murga V, Miguel de la Villa F. Metabolismo óseo y pérdida de masa ósea en los trastornos de la alimentación. An Med Interna (Madrid) 2002; 19: 143-150.

\section{INTRODUCCIÓN}

Los trastornos de la alimentación (anorexia y bulimia nerviosa) afectan aproximadamente al 1-3\% de las mujeres jóvenes adultas y se caracterizan por una restricción intermitente o constante del consumo calórico motivada por una alteración disfuncional en relación al peso, y la forma corporal y la nutrición (1).

Por definición, los pacientes con anorexia nerviosa presentan peso corporal bajo y disfunción gonadal secundaria (amenorrea en mujeres) (1).
Por lo tanto, la amenorrea es uno de los criterios diagnósticos de la anorexia nerviosa, pero no de la bulimia nerviosa (2).

Sin embargo, el $50 \%$ de las mujeres bulímicas pueden presentar irregularidades menstruales (3).

Ambos tipos de enfermedades comparten un síndrome endocrino y metabólico de adaptación al hambre que es bastante similar cualitativamente pero con una intensidad considerablemente menor en la bulimia nerviosa (1).

El ejercicio ha sido definido como un mecanismo que puede "disparar" el desarrollo de la anorexia nerviosa (4); asimis-

Trabajo aceptado: 21 de mayo de 2001

Correspondencia: Mikel Escalante Boleas. C/Gregorio Uzquiano nº 24-1º D. 48920 Portugalete. Bizkaia. 
mo, es un factor significativo que perpetúa los trastornos de la alimentación.

Es un fenómeno bien conocido que la densidad mineral ósea (DMO) disminuída aumenta el riesgo de fracturas patológicas en la anorexia nerviosa (5).

Sin embargo, hay menos información con respecto a la bulimia nerviosa. Así, puede haber DMO normal en el subgrupo de pacientes sin historia de anorexia nerviosa, y disminuída si han tenido dicho antecedente (6) (7) (8).

El riesgo de osteoporosis se ha relacionado con factores genéticos endocrinos, dietéticos (9) y étnicos (10).

El pico de masa ósea se ha definido como la cantidad máxima de hueso que un individuo puede llegar a alcanzar. Va aumentando durante las 2-3 primeras décadas de la vida y llega al máximo entre los 25-30 años, para mantenerse unos 10-15 años antes de comenzar a disminuir (113).

La relación ingesta de calcio/masa ósea continúa hoy en día despertando gran interés científico. Se admite la posibilidad de que una deficiencia de calcio durante la etapa de formación del esqueleto puede contribuir a la obtención de un menor pico de masa ósea, aumentando el riesgo de fracturas en etapas posteriores de la vida (113).

Entre los mecanismos de pérdida ósea se deben considerar: efectos disminuidos de los esteroides gonadales, concentraciones bajas de factores tróficos óseos como el IGF-1 (11), efectos aumentados de los glucocorticoides (12), alta intensidad de ejercicio pero baja de estímulos mecánicos sobre el hueso en relación con el peso corporal (13), consumo disminuido de calcio y otros minerales, y sustancias tóxicas (nicotina, laxantes).

Los efectos negativos del tabaco (14) o el ejercicio (15) pueden ser mediados por un transtorno en la función gonadal.

Los factores clínicos de riesgo para tener una baja DMO incluyen un índice bajo de masa corporal, la larga duración tanto de la anorexia nerviosa como de la amenorrea, y el mal pronóstico de tratamiento global del cuadro (16).

\section{BULIMIA NERVIOSA}

La bulimia nerviosa es un trastorno de la alimentación que puede estar relacionado con la anorexia nerviosa. Así, el 15\% de los pacientes diagnosticados de esta enfermedad habrían sido tratados previamente de anorexia nerviosa $(17,18)$.

Se caracteriza por comportamientos inadecuados de consumo alimenticio excesivo con purga posterior (vómitos, uso de laxantes) y se diagnóstica únicamente en pacientes dentro de un rango de peso normal (7).

Las complicaciones médicas de la bulimia nerviosa pueden incluir disfunción menstrual y amenorrea (19), restricción dietética, hipercortisolismo, anomalías metabólicas y ejercicio excesivo (20). La osteopenia es considerada una complicación asociada en un subgrupo de pacientes con bulimia nerviosa, fundamentalmente en aquéllas con historia antigua de anorexia nerviosa (7).

Algunos autores mantienen que cuanto mayor sea la edad de inicio de la bulimia nerviosa menor parece ser la DMO del paciente (7). Además, la duración de la amenorrea y el índice bajo de masa corporal, son factores a tener en cuenta si las pacientes bulímicas presentan una DMO reducida (7).

Por otro lado, las bulímicas que se ejercitan regular o periódicamente pueden presentar DMO lumbares más altas que las bulímicas sedentarias y las anoréxicas (21).
Estas diferencias según Koch et al, son debidas a un mayor efecto deletéreo de la deprivación nutricional sobre el hueso más que al de la duración de la amenorrea o a los niveles séricos de hormonas sexuales (22). Finalmente, la diferencia en consumo de nutrientes y de energía podría explicar, al menos en parte, porqué las enfermas bulímicas que realizan ejercicio físico, en contraste con las anoréxicas, pueden experimentar un efecto positivo de dicho ejercicio sobre la masa ósea (23).

\section{ANOREXIA NERVIOSA}

La anorexia nerviosa (AN) es un trastorno psiquiátrico caracterizado por malnutrición severa y disfunción hormonal (24).

La deficiencia calórico-protéica y un consumo inadecuado de calcio se acompañan frecuentemente de comportamientos como vómitos autoinducidos, abuso de diuréticos y laxantes y ejercicio compulsivo que culmina en el agotamiento progresivo de los depósitos grasos y de la masa magra corporal (25). Además, son habituales la negativa a mantener el peso corporal en un $85 \%$ o más del peso normal, un intenso miedo de ganar peso, y amenorrea de al menos 3 meses de duración (26).

La prevalencia de la $\mathrm{AN}$ es del $1-4 \%$ entre las niñas en edad escolar y las adolescentes y parece estar aumentando. De ellas, sólo un $50 \%$ se recuperarán por completo (26).

\section{CRITERIOS DIAGNÓSTICOS DE ANOREXIA NERVIOSA (27)}

1. Pérdida del $20 \%$ del peso normal que dura más de 3 meses.

2. Comportamiento alimenticio anormal incluyendo restricción de comida y episodios bulímicos.

3. Trastorno en el modo de percepción de la imagen o el peso corporal propios e intenso miedo de ganar peso, incluso aunque se esté con un peso bajo.

4. Inicio por debajo de los 30 años de edad.

5. En mujeres, amenorrea.

6. Negación de la enfermedad incluyendo otros trastornos psiquiátricos que presenten anorexia y pérdida de peso.

\section{CAMBIOS EN LA COMPOSICIÓN CORPORAL EN LA ANOREXIA NERVIOSA}

La composición corporal puede medirse mediante antropometría, densidad corporal, excreción de creatinina urinaria, parámetros de impedancia y densidad mineral ósea (28).

En la forma más frecuente de anorexia, el tejido magro se pierde no sólo del músculo sino también de vísceras como hígado, bazo y riñones (29).

Durante los primeros 4-5 días de deprivación energética existe un marcado balance acuoso negativo $y$, si continúa el ayuno, la tasa de pérdida acuosa disminuye abruptamente y el agua se conserva (30).

La mayoría del agua perdida inicialmente durante el ayuno se origina del líquido extracelular aunque también puedan producirse cambios importantes en el compartimento intracelular. El músculo esquelético se pierde con rapidez y en situa- 
ciones extremas, la pérdida puede ser superior a las 2 terceras partes del tejido muscular esquelético (31).

La masa muscular de las extremidades superiores tiende a atrofiarse algo más rapidamente que la de las extremidades inferiores o la masa muscular total (32).

Cheek y cols. afirmaron que la reducción en la masa muscular se debe más a una disminución en el tamaño y la masa celulares que a diferencias en el número de células (33). Wang y cols observaron que, si el rango de excreción de creatinina urinaria oscila entre 1-2 g/día, el cociente masa muscular/creatinina disminuye un $10 \%$. El uso de esta aproximación parece ser la forma más adecuada de calcular la masa muscular (34).

Las alteraciones bioeléctricas suelen ser evidentes en sujetos con un índice de masa corporal (IMC) muy bajo (menor de $\left.12 \mathrm{~kg} / \mathrm{m}^{2}\right)(28)$.

Finalmente, se considera que la hidratación de las anoréxicas es anormal sólo si el IMC es menor de $14 \mathrm{~kg} / \mathrm{m}^{2}$ (35).

\section{DENSIDAD MINERAL ÓSEA Y ANOREXIA NERVIOSA}

\section{Formación y reabsorción óseas}

La desmineralización se produce cuando la reabsorción sobrepasa a la formación de hueso, lo que lleva al desarrollo de osteopenia, osteoporosis secundaria y a un mayor riesgo de fracturas patológicas (25).

La osteopenia es función de dos procesos independientes mediados por esteroides: aposición subperióstica (T) y reabsorción medular (m). T continúa aumentando a lo largo de la vida mientras que m es mucho más compleja y consta de reabsorción juvenil $\left(\downarrow_{\mathrm{m}}\right)$, oposición puberal $\left(\downarrow_{\mathrm{m}}\right)$ y reabsorción progresiva en la edad adulta ( $\uparrow \mathrm{m})(36)$.

Como resultado de un moderado aumento de $\mathrm{M}$ y un descenso mucho más pronunciado de $\mathrm{T}$, se produce osteopenia, que no es la reabsorción ósea activa de las osteoporosis posmenopáusicas, sino un fracaso en llevar a cabo la aposición puberal normal del hueso cortical (36).

Calcio y fósforo se regulan controlando su absorción y excreción principalmente a través de las acciones de la PTH, $1,25(\mathrm{OH})_{2} \mathrm{D}$ y calcitonina. La secreción de PTH aumenta al disminuir los niveles de $\mathrm{Ca}$ (37).

El 1,25 $(\mathrm{OH})_{2} \mathrm{D}$ se produce en la piel por exposición a la luz UV y estimula directamente la absorción intestinal de Ca y $\mathrm{P}$ y la síntesis de colágeno, fosfatasa alcalina y osteocalcina en los osteoblastos (37).

La calcitonina previene la reabsorción ósea disminuyendo la reabsorción de $\mathrm{Ca}$ del hueso y aumentando su excreción renal (37).

Los estrógenos pueden facilitar el mantenimiento del hueso inhibiendo la secreción de los promotores de la reabsorción ósea (37).

Los estrógenos pueden facilitar el mantenimiento del hueso inhibiendo la secreción de los promotores de la reabsorción ósea (37).

A pesar de lo mencionado, el mecanismo específico de pérdida ósea en la AN todavía se mantiene desconocido.

Hay pocos estudios en los que se hayan realizado biopsias óseas transiliacas (38). En dichos estudios se ha demostrado tanto OP de recambio bajo (38) como de recambio alto (39).

\section{Anorexia nerviosa y $\mathrm{DMO}$}

La absortimetría fotónica (a.f.) sirve para obtener una medición más precisa de la DMO (37).

La a.f. simple se suele usar para mediciones en el esqueleto apendicular (hueso cortical de brazos y piernas) (40).

La a.f. dual, en cambio, mide la DMO en el esqueleto axial (hueso trabecular) (40).

Se considera que la columna lumbar puede mostrar mejor los efectos de la AN sobre el tejido óseo (41) ya que las enfermedades metabólicas óseas afectan típicamente a regiones con un alto contenido de hueso trabecular, debido a que el turnover óseo es mayor (42).

Cuando se produce perforación o fragmentación en el hueso trabecular, la pérdida ósea puede ser irreversible. Así, las anoréxicas pueden experimentar algún grado de pérdida ósea irreversible en las regiones trabeculares a pesar de que se mejore su estado nutricional $(43,44)$. En realidad, no se conoce a qué velocidad, o en qué grado y porcentaje se recupera la DMO perdida al iniciar la rehabilitación nutricional $(26,37)$.

Otros trabajos concluyen que aquellas mujeres que disminuyen la ingesta de calcio desde la adolescencia presentan una DMO femoral menor que las que siguen una dieta equilibrada en productos lácteos (114).

Sin embargo, hay estudios que afirman que la masa ósea de las anoréxicas disminuye entre la $3^{\mathrm{a}}$ y $4^{\mathrm{a}}$ décadas cuando lo normal es que este descenso se produzca después de los 50 años (49).

Se piensa que el grado de pérdida ósea es considerablemente mayor en la AN que el que acontece en las mujeres posmenopáusicas y aparece muy precozmente en este transtorno (46).

\section{Anorexia nerviosa y fracturas óseas}

Las fracturas osteoporóticas en la AN suelen aparecer después de los 30 años de edad, una vez que las pacientes ya no precisan una atención médica para su enfermedad (47).

A pesar de que la pérdida ósea asociada a la enfermedad es rápida (6-8\% por año según algunos informes y 4-10\% según otros) $(48,49)$ y de la frecuencia de formas crónicas o recurrentes (50-70\% de los casos) (50), el número de fracturas osteoporóticas conocidas en relación con la AN es sorprendentemente bajo (51). Si la recuperación de la enfermedad no se acompaña de una restauración de la masa ósea, un episodio de este transtorno sufrido durante la juventud puede alterar permanentemente la integridad del esqueleto derivando en fracturas óseas (16).

\section{Patofisiología de la desmineralización ósea en la anorexia nerviosa}

La desmineralización ósea que padecen las mujeres con AN se ha atribuido a distintos factores.

Según la literatura, los mayores predictores de pérdida de masa ósea en este colectivo suelen ser la duración de la enfermedad, la duración de la amenorrea y el inadecuado consumo de calcio en la adolescencia.

Así, algunos autores atribuyen la desmineralización ósea a la duración de la $\mathrm{AN}(27,52)$, considerando menos importante 
la duración de la amenorrea y viceversa $(53,54,55)$. Los principales factores implicados son:

-Amenorrea y estrógenos

Existe poca evidencia de cuáles son los efectos directos de los estrógenos sobre el hueso (37).

Parece ser que lo más importante no es el nivel absoluto de estrógenos sino, como ya se ha mencionado, el tiempo que estas hormonas permanecen disminudas (18).

Un valor de estrógenos de $60 \mathrm{ng} / \mathrm{ml}$ puede ser el mínimo requerido para proteger el hueso (56).

Las anomalías de las funciones endocrina e hipotalámica están bien documentadas en la AN (57). Los niveles decendidos de estrógenos se atribuyen más a la restricción de alimento que a un transtorno hipotalámico primario (58).

Así, la restricción alimentaria puede ocasionar una disfunción hipotalámica duradera (59). Por ejemplo, la secreción de vasopresina es anormal y parece persistir tras la recuperación del peso (60).

Por otro lado, la amenorrea puede no sólo indicar un déficit estrogénico, sino también reflejar un defecto en la hormona luteinizante necesaria para inducir la ovulación (61).

-Metabolismo Calcio-Fósforo (Ca-P)

Aunque el consumo diario pueda ser normal, los niveles disminuidos de estrógenos que presentan las pacientes anoréxicas son capaces de reducir la eficiencia de la absorción de calcio (37). No obstante, los niveles séricos de los iones Ca y P suelen ser normales $(62,63)$ aunque haya estudios que describan niveles de $\mathrm{P}$ ligeramente más bajos en este colectivo $(5,64)$.

La excreción de $\mathrm{Ca}$ suele aumentar debido principalmente al incremento en el Ca liberado desde el hueso (64).

La formación y mineralización óseas pueden estar marcadamente alteradas, ocasionando niveles bajos de osteocalcina $(65,66)$.

El P urinario tiende a aumentar a consecuencia del déficit de proteínas en la dieta (67) aunque dicha excreción también puede ser normal $(38,68)$.

\section{-Actividad física}

El ejercicio es fundamental para el mantenimiento y desarrollo de la masa ósea (69). Sin embargo, la ausencia de hormonas sexuales circulantes puede limitar sus efectos positivos sobre la DMO (70).

Así, las atletas amenorréicas suelen presentar DMO más bajas que las mujeres con ciclos menstruales normales (71) (72).

La edad de inicio de la actividad física es importante. El inicio precoz del entrenamiento parece contribuir a una DMO reducida en estas atletas, retrasando o enlenteciendo la maduración del hueso de forma similar a la de las anoréxicas (73).

El peso corporal y el IMC suelen correlacionarse positivamente con la DMO en pacientes anoréxicas $(8,74)$. Por tanto, un peso corporal extremadamente bajo atenuaría los beneficios potenciales del ejercicio sobre la mineralización ósea en estas mujeres (37).

\section{-PTH y fosfatasa alcalina (PALC)}

Los índices fisiológicos de función ósea, como por ejemplo los niveles séricos de PTH y PALC, suelen ser normales en la AN $(37,75)$.

Sin embargo, en algún estudio se ha encontrado que la PALC aumenta paralelamente a la ganancia de peso, lo que sería atribuible a la reanudación del crecimiento óseo (76).

\section{- 1,25 Dihidroxivitamina $D$}

El metabolismo de la vitamina D varía con frecuencia en los transtornos de la alimentación ya que puede verse afectado por un reducido consumo dietético (63), la capacidad disminuida en los receptores de la vitamina $\mathrm{D}$ en pacientes con déficit estrogénico (78) o por la exposición a la luz solar (79).

La mayoría de los estudios revelan niveles normales de vitamina D $(13,68,69,80)$, aunque hay algunos que han demostrado un descenso en los niveles de esta vitamina (81).

\section{- Aclaramiento de creatinina}

El aclaramiento de creatinina es más bajo de forma significativa en mujeres anoréxicas según algunos investigadores. Este hecho puede explicarse por su menor masa muscular y por un IMC disminuido $(76,81,82)$.

-Exceso de glucocorticoides

Se han descrito niveles elevados de cortisol sérico y urinario en la AN $(12,37,74,83,84,85)$.

La mayoría de los estudios atribuyen este hecho a un aumento en la actividad del eje hipotálamo-hipófisis-adrenal (12) y al incremento en la producción con disminución del aclaramiento metabólico del cortisol $(83,86)$.

Varios autores han indicado que los niveles de cortisol disminuyen gradualmente durante la realimentación y vuelven a la normalidad tras la recuperación del peso (74).

Estos hallazgos sugieren que el hipercortisolismo juega un rol a tener en cuenta en la DMO reducida observada en la AN (87).

\section{- Hormona del crecimiento $(\mathrm{GH})$}

Existen varios estudios que propugnan niveles excesivos de $\mathrm{GH}$ en pacientes anoréxicas $(88,89)$.

Sin embargo las acciones de esta hormona no son directas sino mediadas por las somatomedinas que están disminuidas en la AN (37); lo que podría deberse o a una secreción menor de GH o a la alteración de la estimulación de la GH sobre la liberación de somatomedina $C$ hepática $(90,91)$. Los efectos de la $\mathrm{Sm}-\mathrm{C}$ disminuida se pueden modular por numerosos cambios en las proteínas ligadoras de IGF-1 (92).

-Nuevos marcadores del turnover óseo

Se han desarrollado nuevos marcadores del turnover óseo que permiten investigar los mecanismos específicos de la pérdida ósea asociada a la AN. El propéptido carboxi-terminal del procolágeno I (PICP) es una medida de formación ósea y las piridinolinas (desoxipiridinolina) y los puentes del telopéptido carboxi-terminal sérico (ICTP) son marcadores de reabsorción ósea (93).

La osteopenia de la AN parece ser de turnover bajo con un aumento de la reabsorción ósea (incremento de ICTP) y desoxipiridinolina) sin formación ósea concomitante (PALC osteocalcina y PICP inalterados) (93).

\section{TRATAMIENTOS RECOMENDADOS}

Las alternativas de tratamiento deben basarse en parámetros bioquímicos y resultados densitométricos (75).

El reconocimiento del riesgo de osteoporosis es un componente esencial del plan de tratamiento para la AN (26).

Por eso es esencial detectar precozmente la $\mathrm{AN}$, de forma que con la densitometría y el tratamiento oportuno se pueda prevenir el riesgo, habitualmente subestimado, de fracturas osteoporóticas (94).

\section{Rehabilitación nutricional}

Según diferentes autores sólo la rehabilitación nutricional ha conseguido hasta la fecha revertir la pérdida ósea en estas 
pacientes $(1,75)$. Otros trabajos informan que se precisa no sólo la recuperación del peso sino también la reanudación de las menstruaciones para lograr un aumento en la DMO en esta enfermedad $(27,95)$. Asímismo, los déficits del metabolismo mineral adquiridos durante la adolescencia pueden no ser del todo reversibles (27).

La rehabilitación nutricional lleva a un aumento significativo en el peso corporal, lo que implica aumentos en la grasa corporal total, masa magra total y en el contenido mineral óseo, siendo la grasa corporal total la que experimenta un mayor incremento (96).

Las mujeres realimentadas suelen presentar un mayor acúmulo de grasa en las regiones centrales (tórax, abdomen, cadera) que en las extremidades (96).

A pesar del aumento significativo de grasa, las pacientes mantienen la distribución ginecoide de la grasa tras el incremento de peso. La mayor grasa corporal permitirá superar el riesgo potencial de enfermedad cardiovascular a largo plazo (96).

\section{Suplementos de calcio}

El déficit de $\mathrm{Ca}$ se debe probablemente tanto al escaso consumo como a un exceso en su excreción renal por lo que debe considerarse el tto. suplementario (75).

El consumo elevado de fibra ocasiona una peor absorción de $\mathrm{Ca}$ al disminuir su utilización, fundamentalmente a causa de los fitatos (97). El tratamiento consistirá tanto en comidas ricas en $\mathrm{Ca}$ (75) como en dosis orales de unos $800 \mathrm{mg}$ de $\mathrm{Ca}$ elemental (98).

Además, una ligera suplementación de vitamina D combinada con Ca podría ayudar a mejorar la DMO en estas pacientes (99).

\section{Ejercicio}

Un programa de ejercicio equilibrado e individualizado puede ser beneficioso en la AN (75).

Los ejercicios de resistencia y tracción intensos son útiles para aumentar la DMO, ya que a diferencia de la actividad aeróbica, consumen menos energía (99).

En este sentido varios estudios sugieren que un alto nivel de actividad física protege contra la pérdida ósea en pacientes con AN $(100,101)$.

\section{Tratamiento hormonal}

El tratamiento hormonal suele prescribirse si los niveles séricos de estrógenos son bajos (75).

La terapia estrogénica debería considerarse en aquellas pacientes jóvenes de alto riesgo una vez que han alcanzado una estatura adecuada. Los factores de riesgo serían: duración de la amenorrea superior a 36 meses, retraso puberal según el estadiaje de Tanner y los valores de dehidroepiandrosterona sérica, inicio precoz de la amenorrea ( $<13$ años) y evidencia radiológica significativa de osteopenia (36).

Sin embargo algunos estudios han indicado que el tratamiento estrogénico no es capaz de prevenir la osteoporosis progresiva en estas pacientes salvo en aquellas con desnutrición severa (menos del 70\% del peso ideal) (102).

La respuesta al tto. varía segun el tipo, dosis y frecuencia en la administración de los estrógenos $(103,104)$.

Así, el cuello femoral (predominantemente hueso cortical) responde de forma diferente a los estrógenos que la columna vertebral (hueso trabecular) ya que ésta suele experimentar un mayor incremento en la DMO.

Hasta la fecha, el tratamiento sustitutivo estrogénico con contraceptivos orales no parece mejorar la DMO en adolescentes y mujeres jóvenes con AN (103-105).

Los estrógenos orales reducen la sístesis hepática de IGF-1 e incrementan los niveles de GH, mientras que la administración transdérmica aumenta los niveles de IGF-1 sin modificar las concentraciones de GH (hormona del crecimiento) $(106,107)$. Además, mientras la administración oral parece suprimir la osteocalcina y el procolágeno I, la vía transdérmica aumenta dichos parámetros, lo que sugiere un efecto positivo de los estrógenos transdérmicos sobre la función osteoblástica $(108,109)$.

\section{Tratamiento antidepresivo}

El hipercortisolismo aparece a menudo en pacientes depresivos, por lo que el tratamiento primario antidepresivo puede mejorar directamente la DMO. Así, el alprazolam inhibe de forma directa la liberación excesiva de ACTH en mujeres con anovulación en relación al estrés, reduciendo por tanto los niveles de cortisol, y contribuyendo a recuperar la función ovulatoria normal (110).

\section{Flúor}

La administración de fluor suele reservarse para los casos con DMO más disminuida. Las dosis que se requieren son bajas (unos 11,5 mg/día) y pueden provocar aumentos sustanciales en el hueso trabecular (49). Dosis más altas de flúor podrían provocar toxicidad y fracturas de estrés (49).

El tratamiento puede ser igualmente efectivo combinando pequeñas dosis de flúor con hormonas (estrógenos.progesterona)(48).

\section{Bifosfonatos}

El tratamiento con bifosfonatos, según algunos estudios, es efectivo para prevenir la pérdida ósea en mujeres anoréxicas en tratamiento con nutrición parenteral, fundamentalmente en el hueso trabecular (111). No obstante se precisan nuevos estudios que permitan corroborar los hallazgos mencionados.

\section{Nuevos tratamientos potenciales}

El IGF-1 recombinante humano, aplicado vía subutánea durante 6 días, a dosis de $30 \mathrm{ug} / \mathrm{Kg} 2$ veces al día, podría incrementar la formación ósea sin alterar la reabsorción ósea mientras que a dosis más altas aumenta también dicha reabsorción (112).

Estos resultados pueden dar lugar a una nueva terapia potencial aunque el modo de aplicación y las pequeñas dosis terapéuticas limitan su uso (94). 


\section{CONSIDERACIONES FINALES}

En ocasiones, la AN puede pasar desapercibida, bien porque se oculta de forma voluntaria, bien debido a un diagnóstico erróneo.

Es necesario investigar la existencia de esta enfermedad en mujeres que tengan un peso ligeramente descendido, ya que los transtornos del comportamiento alimenticio y de la menstruación no se suelen revelar de forma espontánea por parte de estas personas.
Gracias a la valoración exacta del IMC, a un interrogatorio preciso sobre el estado hormonal (actual y pasado) y a los criterios diagnósticos de la AN, puede establecerse el diagnóstico.

La osteoporosis de la AN es probablemente frecuente y mal conocida, sobre todo en mujeres no menopaúsicas y su mejor conocimiento debería permitir realizar un diagnóstico más preciso y precoz.

\section{Bibliografía}

1. Goebel G, Schweiger U, Krüger R, Fichter MM. Predictors of bone mineral density in patients with eating disorders. Int J Eat Disord 1999; 25: $143-50$

2. American Psychiatric Association. 1994 Diagnostic and statistical manual of mental disorders, 4 th ed. Washington DC: American Psychiatric Association.

3. Pirke K, Schweiger M, Laersle U, Dickhaut R. Dieting influences the menstrual cycle: vegetarian vs non vegetarian diet. Fertil Steril 1986; 46: 1083-8.

4. Epling WF, Pierce WD, Stefan A. A theory of activity based anorexia. Int J Eat Disord 1983; 3: 27-46.

5. Brotman A, Stern TA. Osteoporosis and pathological fractures in anorexia nervosa. Am J Phychiatry 1985; 142: 495-6.

6. Joyce J, Warren DL, Humphries LL, Smith AJ, Coon JS. Osteoporosis in women with eating disorders: comparison of physical parameters, exercise and menstrual status wirh SPA and DPA evaluation. J Nucl Med 1994; 31: 325-31.

7. Newton JR, Freeman CP, Hannan WJ, Cowen S. Osteoporosis and normal weight bulimia nervosa-which patients are at risk?. J Psychosomatic Res 1993; 37: 239-47.

8. Davies KM, Pearson PH, Huseman CA, Greger NG, Kimmel DK, Recker RR. Reduced bone mineral in patients with eating disorders. Bone 1990; 11: 143-7.

9. Cummings SR, Kelsey JL, Nevitt MC, O’ Dowd KJ. Epidemiology of osteoporosis and osteoporotic fractures. Epidemiol Rev 1985; 7: 178-208.

10. Pollitzer WS, Anderson JJB. Ethnic and genetic differences in bone mass: a review with a hereditary vs environmental perspective. Am J Clin Nutr 1989; 50: 1244-59.

11. Kelly PJ, Eisman JA, Stuart MC, Pocock NA, Sambrook PN, Gwin TH Somatomedin-C, physical fitness and bone density. J Clin Endocrinol Metab 1990; 70: 718-23

12. Newman MM, Halmi KA. Relationship of bone density to estradiol ande cortisol in anorexia nervosa and bulimia. Psychiat Res 1989; 29: 105-12.

13. Rigotti NA, Nussbaum SR, Herzog DB, Neer RM. Osteoporosis in women with anorexia nervosa. N Engl J Med 1984; 311: 1601-6.

14. Hopper JL, Seeman E. The bone density of female twins discordant for tobacco use. N Engl J Med 1994; 330: 387-92.

15. Hetland HL, Haarbo J, Christiansen C, Larsen T. Running induces menstrual disturbances but bone mass is unaffected, except in amenorrheic women. Am J Med 1993; 95: 53-60.

16. Rigotti NA, Neer RM, Skates SJ, Herzog DB, Nussbaun SR. The clinical course of osteoporosis in anorexia nervosa. JAMA 1991; 265: 1133-8.

17. Turnbull J, Freeman CP, Barrie F, Henderson A. The clinical characteristics of bulimic women. Int J Eat Disord 1989; 8: 399-409.

18. Ryle RL, Mitchell JE, Eckert ED. Bulimia: report of 31 cases. J Clin Psychiat 1981; 42: 60-4.

19. Devlin MJ, Walsh BT, Roose SP, Katz J, Linkie D. Gonadotrophin patterns in eating disorders. Psychiat Resp 1989; 28: 11-24.

20. Mitchell JE, Seim HC, Colon E, Pomeroy C. Medical complication and medical management of bulimia. Ann Intern Med 1987; 107: 71-7.

21. Sundgot-Borgen J, Bahr R, Falch JA, Sundgot Scheneider L. Normal bone mass in bulimic women. J Clin Endocrinol Metab 1988; 83: 3144-9.

22. Koch SW, Noriega E, Leslie K, Muller C, Harvison JE. Bone mass and soft tissue composition in adolescent with anorexia nervosa. Bone 1996; 19: $181-8$
23. Sundgot Borgen J. Nutrient intake of female elite athletes suffering from eating disorders. Int J Sport Nutr 1993; 3: 431-42.

24. Hsu LKG. Eating disorders. 1990. New York: Guilford Press

25. Siemers B, Chakmakjian Z, Gench B. Bone density patterns in women with anorexia nervosa. Int J Eat Disord 1996; 19 (2): 179-86.

26. Mehler PS. Bone density in amenorrheic athletes and in anorexia nervosa. JAMA 1996; 276 (17): 1384-5.

27. Hotta M, Shibasaki T, Sato K, Damura H. The importance of body weight history in the occurrence and recovery of osteoporosis in patients with anorexia nervosa: evaluation by dual X-ray absorptiometry and bone metabolic markers. Eur J Endocrinol 1998; 139: 27683.

28. Polito A, Cuzzolaro M, Raguzzini A, Censi L, Ferro-Luzzi A. Body composition changes in anorexia nervosa. Eur J Clin Nutr 1988; 52 655-62.

29. Fohlin L. Body composition cardiovascular and renal function in adolescent patients with anorexia nervosa. Acta Ped Scand 1977; 268: 7-20

30. Drenick EJ. The effects of acute and prolonged fasting and refeeding on water, electrolyte and acid-base metabolism. In Clinical Disorders of Fluid and Electrolyte Metabolism, eds. MH Maxwell \& CR Kleeman. New York: Mc Graw-Hill Book Company.

31. Heymsfield SB, Olafson RP, Kunter MH, Nixon DW. A radiographic method of quantifying protein-calorie under nutrition. Am J Clin Nutr 1979; 32: 693-702.

32. Keys A, Brozek J, Henschel A, Taylor HL. The biology of human starvation. Minneapolis: University of Minnesota Press 1950.

33. Cheek DB, Hill DE, Cordano A, Graham GC. Malnutrion in infancy: changes in muscle and adipose tissue before and after rehabilitation. Ped Res 1970; 4: 135-44.

34. Wang ZM, Gallagher D, Nelson ME, Mathews DE, Heymsfield SB Total body skeletal muscle mass: evaluation of 24-h urinary creatinine excretion by computarized axial tomography. Am J Clin Nutr 1996; 63: $863-9$

35. Hannan J, Cowen S, Freeman C, Mackie A, Shapiro CM. Assessment of body composition in anorexic patients. In: In vivo Body Composition Study Recent Advances, eds. S. Yasumura, JE Harrison, KG Mc Niel, AV Woodhead and FA Dilmanian, pp 149-55. Plenum Press: New York.

36. Ayers JWT, Gidwani GP, Schmidt IMV, Gross M. Osteopenia in hypoestrogenic young women with anorexia nervosa. Fertil Steril 1984; 41 (2): 224-8.

37. Salisbury JJ, Mitchell JE. Bone mineral density and anorexia nervosa in women. Am J Psychiatry 1991; 148: 768-774.

38. Kaplan FS, Petschuk MJ, Fallon M, Haddad J. Osteoporosis and hip fracture in a young woman with anorexia nervosa. Clin Orthop 1986 212: 250-4.

39. Joyce JM, Warren DL, Humphries LL, Smith AJ, Coon JS. Osteoporosis in women with eating disorders: comparison of physical parameters, exercise, and menstrual status with SPA and DPA evaluation. J Nucl Med 1990; 31: 325-31

40. Wahner H, Dunn W, Riggs B. Noninvasive bone mineral measurements. Semin Nucl Med 1983; 13: 282-289.

41. Mazess R, Barden H, Oherich E. Skeletal and body composition effects of anorexia nervosa. Am J Clin Nutr 1990; 11: 143-147.

42. Seeman E, Wahner HW, Oxford KP, Kumar R, Johnson WJ, Riggs BL. Differential effects of endocrine dysfunction on the axial and the apprendicular skeleton. J Clin Invest 1982; 69: 1302-13. 
43. Mosekilde L, Eriksen EF, Charles P. Effects of thyroid hormones on bone mineral metabolism. Endocrinol and Met Clin North America 1990; 11: 35-63.

44. Baum ML, Kramer EL, Sanger JJ, Pena A. Stress fractures and reduced bone mineral density with prior anorexia nervosa. J Nucl Med 1987; 28 (9): 1506-7.

45. Passloff ES, Slap GB, Pertschuk MJ, Attie MF, Kaplan FS. A longitudinal study of metacarpal bone morphometry in anorexia nervosa. Clin Orthop Rel Res 1992; 278: 217-25.

46. Armstrong V. Effects of anorexia nervosa on bone density. British Journal of General Practice 1995; 387

47. Pape HJ, Hudson JJ, Yurgelun-Todd D. Prevalence of anorexia nervosa and bulimia in three student populations. Int J Eat Disord 1984; 3: 45.

48. Maugars Y, Prost A. Osteoporose de l'anorexia mentale. Presse Med 1994; 24: 156-8.

49. Maugars Y, Berthelot JM, Forestier R et al. Follow-up of bone mineral density in 27 cases of anorexia nervosa. Eur J Endocrinol 1996; 135: 591-7.

50. Hsu LKG, Crisp AH, Harding B. Outcome of anorexia nervosa. Lancet 1979; 1: 61.

51. Maugars Y, Berthelot JM, Lalande S, Charlier C, Prost A. Osteoporosis fractures revealing anorexia nervosa in five females. Rev Rhum (Engl Ed) 1996; 63 (3): 201-6.

52. Hay P. Bone loss in patients with anorexia nervosa. Am J Psychiatry 1992; 149(3): 415-6

53. Ward A, Brown N, Treasure J. Persistent osteopenia after recovery from anorexia nerviosa. Eat Disord 1997; 22: 71-5.

54. Brue T, Chabert-Orsini V, Harlik P, Poet JL, Conte-Devolx B. Evaluation de la masse osseuse vertebrale dans l' anorexie mentale. Annales d' Endocrinologie (Paris) 1994; 54: 175-9.

55. Poet JL, Galinier Pujol A, Tonolli-Serabian I, Conte-Devolx B, Roux H. Lumbar bone mineral density in anorexia nervosa. Clin Rheum 1993; 12(2): 236-9.

56. Mc Intyre I. Therapeutic levels of estradiol in postmenopausal women. Lecture in symposium new perspective on hormone replacement therapy. Boston, April 30, 1988.

57. Newman M, Halmi K. The endocrinology of anorexia nervosa and bulimia nervosa in The Endocrinology of Eating Disorders. Edited by Brown WA. Philadelphia, WB Saunders, 1988.

58. Philipp E, Schweiger V, Spyra B et al. Effects of different forms of malnutrition on central neurotransmitter activity and neuroendocrine regulations in experimental animals, in Advances in the Biosciencies, vol 60: Disorders of eating Behaviour: A Psychoneuroendocrine Approach. Edited by Ferrari E, Brambilla F. Oxford, Pergamon Press, 1985.

59. Russell G, Loraine J, Bell E et al. Gonadotrophin and estrogen excretion in patients with anorexia nervosa. J Psychosom Res 1965; 9: 79-85.

60. Gold PW, Kaye W, Robertson GL et al. Abnormalities in plasma and cerebrospinal-fluid arginine vasopressin in patients with anorexia nervosa. N Engl J Med 1983; 308: 1117-23.

61. Schweiger V, Pirke KM, Laessle RG, Fichter MM. Gonadotrophin secretion in bulimia nervosa. J Clin Endocrinol Metab 1992; 74:1122-7.

62. Treasure J, Russell J, Fogelman I et al. Reversible bone loss in anorexia nervosa. Br Med 1987; 293: 474-5.

63. Crosby L, Kaplan F, Pertschuk M et al. The effect of anorexia nervosa on bone morphometry in young women. Clin Orthop 1985; 201:271-7.

64. Abrams SA, Silber TJ, Esteban NV et al. Mineral balance and bone turnover in adolescents with anorexia nervosa. J Pediatr 1993: 123: 32631 .

65. Mira M, Stewart PM, Vizzard J, Abraham S. Biochemical abnormalities in anorexia nervosa and bulimia. Ann Clin Biochem 1987, 24: 29-35.

66. Saggesse G, Bertelloni S, Baroncelli GI, Ghirri P, Cosenza GM, Buggiani B. Ormoni calciotropi nell' osteoporosi dell'anoressia nervosa. Minerva Pediatr 1989; 41: 61-5.

67. Orwell E, Ware M, Stribrska L et al. Effects of dietary protein defficiency on mineral metabolism and bone mineral density. Am J Clin Nutr 1992; 56: 314-9.

68. Maugars Y, Prost A. Osteoporose trabeculaire compliquant une anorexia mentale meconnue. Rev Rhum Mal Osteoartic 1989; 56: 159-61.

69. Ott SM. Attainment of peak bone mass (editorial). J Clin Endocrinol Metab 1990; 71: 1082 A-1990.

70. Frost HM. The role of changes in mechanical usage set points in the pathogenesis of osteoporosis. J Bone Miner Res 1992; 7: 253-61.

71. Drinkwater B, Bruemner B, Chesnut C. Menstrual history: a determinant of current bone density in young athletes. JAMA 1990; 263: 545-8.

72. Marcus R, Cann C, Madvig P et al. Menstrual function and bone mass in elite women distance runners. Ann Intern Med 1985; 102: 158-63.
73. Biller B, Saxe V, Herzog D et al. Mechanism of osteoporosis in adult and adolescent women with anorexia nervosa. J Clin Endocrinol Metab 1989; 68: 548-54.

74. Bachrach L, Guido D, Katzman D et al. Decreased bone density in adolescent girls with anorexia nervosa. Pediatrics 1990; 86: 440-7.

75. Carmichael KA, Carmichael DH. Bone metabolism and osteopenia in eating disorders. Medicine 1995; 74: 254-67.

76. Matthews B, Lacey J, Clave H. Premature loss of bone in chronic anorexia nervosa (letter). Br Med J 1985; 290: 1431.

77. Olmos JM, Riancho JA, Amado JA, Freijanes J, Menéndez-Arango J, González Macías J. Vitamin D metabolism and serum binding proteins in anorexia nervosa. Bone 1991; 12: 43-6.

78. Liel Y, Kraus S, Levy J, Shany S. Evidence that estrogens modulate activity and increase the number of 1,25 dihydroxyvitamin $\mathrm{D}$ receptors in osteoblast-like cells. Endocrinology 1992; 130: 2597-601.

79. Fonseca V, D' Souza V, Houlder S et al. Vitamin D deficiency and low osteocalcin concentrations in anorexia nervosa. J Clin Pathol 1988; 41 : 195-7.

80. Mc Anarney ER, Greydanus DE, Campanella VA, Hoekelman RA. Rib fractures and anorexia nervosa. J Adolesc Health Care 1983; 4: 40-3.

81. Brion LP, Boeck MA, Gauthier B, Nussbaum MP, Schwartz GJ. Estimation of glomerular filtration rate in anorectic adolescents. Pediatr Nephrol 1989; 3:16-21.

82. Yendt ER, Cohanim M, Jarzylo S, Jones G, Rosenberg G. Bone mass is related to creatinine clearance in normal elderly women. J Bone Miner Res 1991; 6: 1043-50.

83. Boyar RM, Hellman LD, Roffwarg $\mathrm{H}$ et al. Cortisol secretion and metabolism in anorexia nervosa. N Engl J Med 1977; 296: 190-3.

84. Gold PW, Gwirtsman H, Avgerinos PC et al. Abnormal hypothalamic pituitary-adrenal function in anorexia nervosa. N Engl J Med 1986; 314: 1335-42.

85. Larocca FEF. Concurrence of Turner' s syndrome, anorexia nervosa and mood disorders: Case report. J Clin Psychiatry 1985; 46: 296-7.

86. Casper R, Chatterton R, Davis J. Alterations in serum cortisol and its binding characteristics in anorexia nervosa. J Clin Endocrinol Metab 1979; 49: 406-11.

87. Waslh B, Katz J, Levin J et al. The production rate of cortisol declines during recovery from anorexia nervosa. J Clin Endocrinol Metab 1981; 53: 203-5.

88. Rappaport R, Rerot C, Czernichow P. Somatomedin activity and growth hormone secretion, I: Changes related to body weight in anorexia nervosa. Acta Paediatr Scan 1980; 69: 37-41.

89. Tanaka T, Maesaka H, Suwa S. Changes in somatomedin activity in anorexia nervosa. Endocrinol Jpn 1985; 32: 891-7.

90. Andersen AE, Mickalide AD. Anorexia nervosa in the male: an underdiagnosed disorder. Psychosomatics 1983; 24: 1066-75.

91. Rigotti NA, Neer RM, Jameson L. Osteopenia and bone fractures in a man with anorexia nervosa and hypogonadism. JAMA 1986; 256: $385-8$

92. Counts DR, Gwirtsman H, Carlsson LMS, Lesem M, Cutler GB. The effect of anorexia nervosa and refeeding on growth hormona binding protein the insulin-like growth factors (IGFs) and the IGF-binding proteins. J Clin Endocrinol Metab 1992; 75: 762-7.

93. Lennkh C, Zwaan M, Bailer V. Osteopenia in anorexia nervosa: specific mechanisms of bone loss. J Psych Res 1999; 33: 349-56.

94. Maugars Y., Clochon P, Grardel B et al. Etude du retentissement osseux dans 33 cas d' anorexia mentale dont 8 avec une osteoporose fracturaire. Rev Rhum 1991; 58: 751-8.

95. Iketani T, Kiriike N, Nakanishi S, Nakasuji T. Effects of weight gain and resumption of menses on reduced bone density in patients with anorexia nervosa. Biol Psychiatry 1995; 37: 521-7.

96. Orphanidou CI, Mc Cargar LJ, Birmingham CL, Belzberg AS. Changes in body composition and fat distribution after short-term weight gain in patients with anorexia nervosa. Am J Clin Nutr 1997; 65: 1034-41.

97. Pronsky ZM. Powers and Moore's food medication interactions 10th ed. Pottstown, Pa: Food Medication Interactions; 1997.

98. Brooks ER, Howat PM, Cavalier DS. Calcium supplentation and exercise increase appendicular bone density in anorexia: a case study. J Am Diet Assoc 1999; 99: 591-3.

99. Frost HM. A determinant of bone architecture: the minimum effective strain. Clin Orthop 1983; 175: 286-92.

100. Joyce JM, Warren DL, Humphries LL, Smith AJ, Coon JS. Osteoporosis in women with eating disorders: comparison of physical parameters exercise and menstrual status with SPA and DPA evaluation. J Nucl Med 1990; 31: 325-31. 
101. Kiriike N, Iketani T, Nakanishi S et al. Reduced bone density and major hormones regulating calcium metabolism in anorexia nervosa. Acta Psychiat Scand 1992; 86: 358-63.

102. Jones KP, Ravnikar VA, Tulchinsky D, Schiff I. Comparison of bone density in amenorrheic women du to athletics, weight loss, and premature menopause. Obstet Gynecol 1985; 66: 5-8.

103. Klibanski A, Biller BM, Schoenfeld DA, Herzog DB, Saxe VC. The effects of estrogens administration on trabecular bone loss in young women with anorexia nervosa. J Clin Endocrinol Metab 1995; 80: 898-904.

104. Kreipe RE, Hicks DG, Rosier RN et al. Preliminary findings on the effects of sex hormones on bone metabolism in anorexia nervosa. J Adolesc Health 1993; 14: 319-24.

109. Hergenroeder AC. Bone mineralization hypothalamic amenorrhea, and sex steroid therapy in female adolescents and young adults. J Pediatr 1995; 126: 683-9.

106. Kelly J , Rajkovic JA, O' Sullivan AJ et al. Effects of different oral estrogen formulations on insulin-like growth factor I growth hormone and growth hormone binding protein in post-menopausal women. Clin Endocrinol 1993; 39: 561-7.

107. Campagnoli C, Biglia N, Altare F et al. Differential effects of oral conjugated estrogens and transdermal estradiol on insulin-like growth factor I, growth hormone and sex hormone binding globulin serum levels. Gynecol Endocrinol 1993; 7: 251-8.
108. Ho KK, Weissberger AJ. Impact of short-term estrogen administration on growth hormone secretion and action: distinct route-dependent effects on connective and bone tissue metabolism. J Bone Miner Res 1992; 7: 821-7.

109. Harel Z, Riggs S. Transdermal versus oral administration of estrogen in the management of lumbar spine osteopenia in an adolescent with anorexia nervosa. J Adolesc Health 1997; 21: 179-82.

110. Judd SJ, Wong J, Saloniklis S et al The effect of alprazolam on serum cortisol and luteinizing hormone pulsatility in normal women and in women with stress-related anovulation. J Clin Endocrinol Metab 1995; 80: 818-23.

111. Kopp HG, Rüegsegger P, Dambacher MA. Knochensubstanzverlust bei Anorexie-Patientinnen unter Sondenernährung und dessen Prävention mit Bisphosphonaten. Schweiz med Wschr 1992; 122: 538-43.

112. Grinspoon S, Baum J, Howard N et al. Effects of short-term recombinant human insulin-like growh factor 1 administration on bone turnover in osteopenic women with anorexia nervosa. J Clin Endocrinol Metab 1996; 81: 3864-70.

113. Palacios S. Relación entre ingesta de calcio y masa ósea. An Med Interna (Madrid) 1998; 15:61-2.

114. Orozco P, Ruiz Gil E, Nella Sole JM. ¿Hay relación entre los hábitos alimenticios y estilo de vida con la masa ósea en mujeres fértiles?. An Med Interna (Madrid) 1998; 15: 63-9. 